\title{
Methylene Blue Adsorption Study Using Different Zno Types (Normal, Shaheed Factory, Nano)
}

\author{
Khulood Abed Saleh ${ }^{1}$, Hamza Hatem Al-Taie ${ }^{2}$ \\ ${ }^{1,2}$ Department of Chemistry, College of Science, University of Baghdad, Iraq
}

\begin{abstract}
The purpose of this research is to study the adsorption of methylene blue (M.B) on different types of ZnO (normal, shaheed factory copper and brass industry \& nano) at $298 \mathrm{~K} \&$ different $M . B$ concentration, then choose which one is the best for the removal of M.B dye, the adsorption process studies an independent of temperature. The adsorption kinetic was achieved and oby the first order model. The adsorption isotherm for (normal \& shaheed) ZnO was fitting to Freundlich model while nano ZnO was fitting to Temkin isotherm. Its clear that, nano $\mathrm{ZnO}$ is the best then normal $\mathrm{ZnO}$ and shaheed $\mathrm{ZnO}$ is the latest for the removal of M.B depend on \% Removal and particle size. The three types of $\mathrm{ZnO}$ were identify by AFM (Atomic Force Microscope) to measure the particle size and scan its morphology.
\end{abstract}

Keyword: Nano $\mathrm{ZnO}, \mathrm{ZnO}$, AFM, Adsorption, methylene blue

\section{Introduction}

Environmental pollutant is the major cause for most of health illness. Water is the main source of contamination and pollution which effects health through biomagnification. Water gets pollutants from harmful chemicals, dyes, oils etc [1]. The types of water pollution were: (organic, inorganic, biological) pollutants [2]. Methylene blue was also the first synthetic compound ever used as an antiseptic in clinical therapy, and the first antiseptic dye to be used therapeutically. In fact, the use of methylene blue and its derivatives was widespread before the advent of sulfonamides and penicillin [3]. The molecular formula for Methylene blue is $\mathrm{C}_{16} \mathrm{H}_{18} \mathrm{ClN}_{3} \mathrm{~S}$ and its structural is shown in the figure (1):<smiles>CN(C)c1ccc2nc3ccc(=[N+](C)C)cc-3sc2c1</smiles>

Figure 1: Chemical formula of methylene blue

The remove of pollution (i.e.: dyes) are available by current treatment technologies \& can be used (physicochemical, biological) treatment techniques. The physicochemical processes involves (Biological methods [4], enzyme treatment [5], solvent extraction [6], adsorption [7], Advanced oxidation processes [8], electrochemical oxidation [9], catalytic oxidation [10], ion selective electrode [11] etc. Adsorption is the tendency for accumulation of a substance to take place at a surface or at an interface the accummence of adsorption is due to the atoms in any surface being subject to unbalanced forces of attraction perpendicular to the surface plane and therefore possessing certain unsaturation [12].

It is the contact of immiscible phases addition to adsorption, result in penetration of the bulk of one phase by the other [13].Adsorption has traditionally been divided into two kinds (weak physi-sorption, strong chemisorption), physic-sorption occurs when vapors near their saturation pressures adsorb to a dry surface in a process that resembles condensation. The Van der Waals forces and weak dipole interactions present here have heats of adsorption typically a few hundred cal/mol [14].

Chemical adsorption is a much stronger interaction than physical adsorption with heats of adsorption up to $800 \mathrm{~kJ} /$ mole. But heat of adsorption values less than $80 \mathrm{~kJ} / \mathrm{mole}$ do not necessarily rule out chemisorption. During the chemisorption process the adsorbing gas or vapor molecule splits into atoms, radicals, or ions that form a chemical bond with the adsorption site. This interaction involves the sharing of electrons between the gas and the solid surface and may be regarded as the formation of a surface compound [15]. There are many theories that describe the adsorption processes like (Langmuir, Freundlich, Temkin, etc) models. The Langmuir model describes quantitatively the formation of a monolayer adsorbate on the outer surface of the adsorbent, and after that no further adsorption takes place [16]., Langmuir represented by the following equation [17]:

$$
q_{e}=\frac{Q_{0} K_{L} C_{e}}{1+K_{L} C_{e}}
$$

Where: $\mathrm{C}_{\mathrm{e}}=$ the equilibrium concentration of adsorbate $\left(\mathrm{mg} / \mathrm{L}^{-1}\right), q_{e}=$ the amount of pollutant adsorbed per gram of the adsorbent at equilibrium $(\mathrm{mg} / \mathrm{g}), Q_{0}=$ maximum monolayer coverage capacity $(\mathrm{mg} / \mathrm{g}), \mathrm{K}_{\mathrm{L}}=$ Langmuir isotherm constant (L/mg). The Freundlich model commonly used to describe the adsorption characteristics for the heterogeneous surface [18].These data often fit the empirical equation proposed by Freundlich [19]:

$$
\mathrm{Q}_{\mathrm{e}}=\mathrm{K}_{\mathrm{f}} \mathrm{C}_{\mathrm{e}}^{1 / \mathrm{n}}
$$

Where: $\mathrm{K}_{\mathrm{f}}=$ Freundlich isotherm constant $(\mathrm{mg} / \mathrm{g}), \mathrm{n}=$ adsorption intensity; $\mathrm{Ce}=$ the equilibrium concentration of adsorbate $(\mathrm{mg} / \mathrm{L}) Q_{e}=$ the amount of adsorbate adsorbed per gram of the adsorbent at equilibrium $(\mathrm{mg} / \mathrm{g})$. Linearizing Freundlich equation (2), can be written as:

$$
\log Q_{e}=\log K_{f}+1 / n \log C_{e}
$$




\section{International Journal of Science and Research (IJSR) \\ ISSN (Online): 2319-7064}

Index Copernicus Value (2016): 79.57 | Impact Factor (2015): 6.391

The constant $\mathrm{K}_{\mathrm{f}}$ is an approximate indicator of adsorption capacity, while $1 / \mathrm{n}$ is a function of the strength of adsorption in the adsorption process [20].

The Tempkin is other contains a factor that explicitly taking $i$ nto the account of adsorbent-adsorbate interactions. By ignoring the extremely low and large value of concentrations, the model assumes that heat of adsorption (function of temperature) of all molecules in the layer would decrease linearity rather than logarithmic with coverage $[21,22]$.The Temkin model is given by the following equation [21]:

$$
\begin{gathered}
q_{e}=\frac{R T}{b} \ln \left(\mathrm{A}_{\mathrm{T}} \mathrm{C}_{\mathrm{e}}\right) \\
q_{e}=\frac{R T}{b_{T}} \ln \mathrm{A}_{\mathrm{T}}+\left(\frac{R T}{b}\right) \ln \mathrm{C}_{\mathrm{e}} \\
\mathrm{B}=\frac{R T}{b_{T}} \\
q_{e}=\mathrm{B} \ln \mathrm{A}_{\mathrm{T}}+\mathrm{B} \ln \mathrm{C}_{\mathrm{e}}
\end{gathered}
$$

Where:

$A_{T}=$ Temkin isotherm equilibrium binding constant $(\mathrm{L} / \mathrm{g})$, $\mathrm{b}_{\mathrm{T}}=$ Temkin isotherm constant, $\mathrm{R}=$ universal gas constant $\left(8.314 \mathrm{~J} \mathrm{JK}^{-1} \mathrm{~mol}^{-1}\right), \mathrm{T}=$ Temperature at $298 \mathrm{~K}, \mathrm{~B}=$ Constant related to heat of sorption $(\mathrm{J} / \mathrm{mol})$.

\section{Experimental part:}

\section{1- Determination of Maximum Absorption wave length $\left(\lambda_{\max }\right)$ for M.B:}

Wavelength values utilize for estimation of quantity of compound are $664 \mathrm{~nm}$ for Methylene blue as show in (Figure 2).

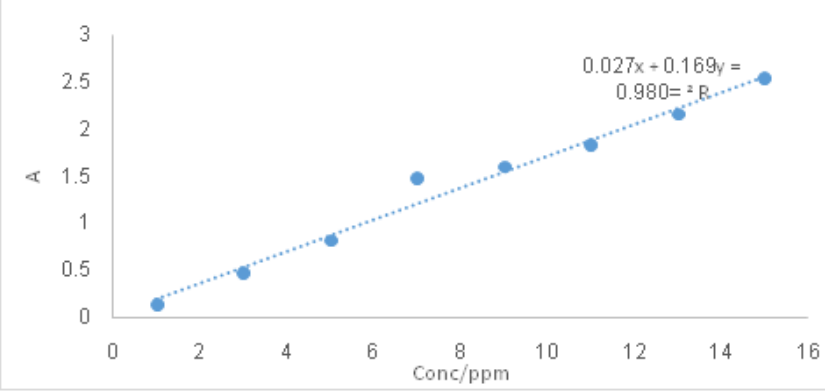

Figure 3: Calibration curve for methylene blue at $\lambda_{\max }=$ $664 \mathrm{~nm}, \mathrm{~T}=298 \mathrm{~K}$

\section{3- Preparation of Methylene blue solutions:}

Six concentrations of Methylene blue (10, 25, 50, 100, 150) $\mathrm{ppm}$ was prepared in $100 \mathrm{ml}$ from stock solution of (1000 ppm M.B) to be used in Adsorption processes.

\section{4- Calculation of $Q_{\mathrm{e}}$ and adsorption isotherm:}

$\mathrm{Q}_{\mathrm{e}}$ can be calculated from the following equation [23]:

$$
\begin{aligned}
& \mathrm{C}_{\mathrm{e}}=\frac{\text { A-Intercept }}{\text { Slope }} \\
& \mathrm{Q}_{\mathrm{e}}=\frac{C_{o}-C_{e}}{W t} * \mathrm{~V}
\end{aligned}
$$

Where: $\mathrm{Q}_{\mathrm{e}}$ : Adsorption capacity of the adsorbent at equilibrium $(\mathrm{mg} / \mathrm{g}), \mathrm{C}_{\mathrm{e}}$ : Equilibrium concentration of adsorbate after adsorption has occurred $(\mathrm{mg} / \mathrm{ml}), \mathrm{C}_{\mathrm{o}}$ : Initial concentration of adsorbate $(\mathrm{mg} / \mathrm{ml}), \mathrm{V}$ : volume of solution (ml).

Adsorption of M.Bdye from an aqueous solution on different ZnOtypes surface were studied at different temperature, The plotted of $\mathrm{Q}_{\mathrm{e}}$ versus equilibrium concentration $\left(\mathrm{C}_{\mathrm{e}}\right)$ gives the type of adsorption isotherm.

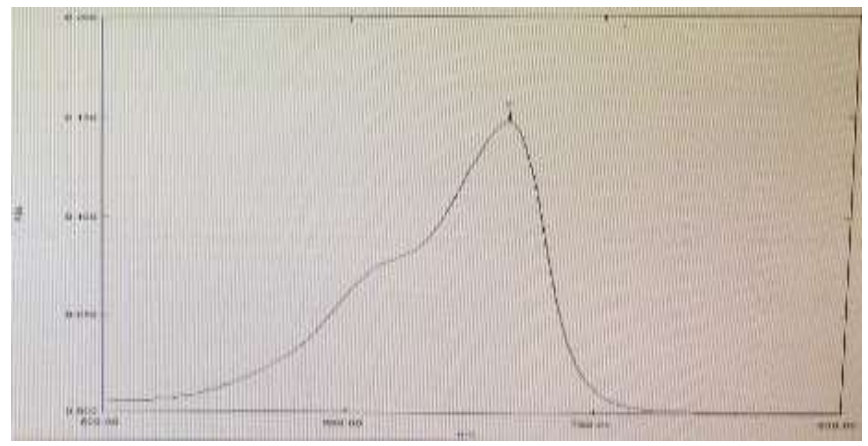

Figure 2: UV-Visible absorption spectrum for Methylene blue dye

\section{2- Calibration Curve:}

Figure 3 show the M.B calibration curve
5- Methylene blue \% removal:

The \% removal was calculated from the following equation [24]:

$$
\% R=\frac{\mathrm{Co}-\mathrm{Ce}}{\mathrm{Co}} * 100
$$

Where:

$\mathrm{C}_{\mathrm{o}}$ : Initial concentration of M.B

$\mathrm{C}_{\mathrm{e}}$ : concentration of M.B after adsorption process

$\%$ R: percentage removal

Volume 6 Issue 12, December 2017 


\section{International Journal of Science and Research (IJSR) \\ ISSN (Online): 2319-7064 \\ Index Copernicus Value (2016): 79.57 | Impact Factor (2015): 6.391}

\section{6- Thermodynamic parameters:}

The standard free energy was determined using the following equation [25]:

$$
\Delta \mathrm{G}_{\mathrm{ads}}^{\mathrm{o}}=-\mathrm{RT} \ln \mathrm{K}
$$

Where: $\Delta \mathrm{G}_{\text {ads }}^{\mathrm{o}}$ : The free energy $\mathrm{kJ} / \mathrm{mol}, \mathrm{T}$ : Absolute temperature in kelven, $\mathrm{R}$ : The gas constant $\left(8.314 \mathrm{JK}^{-1}\right.$ $\mathrm{mol}^{-1}$ ), K: The Langmuir constant, or Freundlish constant, or Tempkin constant, or the thermodynamic equilibrium constant obtained using the method of Khan \& Singh.

The thermodynamic equilibrium constant (K) can be calculated also from the following equation [26]:

$$
K=\frac{a_{s}}{a_{e}}=\frac{v_{s} Q_{e}}{v_{e} C_{e}}
$$

Where: $a_{s}$ : The activity of dye in solid phase, $a_{e}$ : The activity of dye in solution at equilibrium, $v_{s}$ : The activity coefficient of the absorbed dye, $v_{e}$ : The activity coefficient of the dye in solution at equilibrium. The other thermodynamic parameters such as change in standard entropy $\Delta \mathrm{S}^{\mathrm{o}}$ \& standared enthalpy $\Delta \mathrm{H}^{\mathrm{o}}$ were estimated from the following equation:

$$
\ln \mathrm{K}=\frac{\Delta \mathrm{S}}{R}-\frac{\Delta \mathrm{H}}{R} \frac{1}{T}
$$

The value of change enthalpy $\Delta \mathrm{H}^{\circ} \&$ entropy $\Delta \mathrm{S}^{\circ}$ were determined from the slope $\&$ intercept of the plot of $\operatorname{lnKvs}$ $(1 / \mathrm{T})$ respectively.

\section{Result and Discussion}

\section{1- $\quad$ Characterization of $\mathrm{ZnO}$ :}

The AFM analysis provides the measurements of average grain size (and the granularity cumulating distribution for normal $\mathrm{ZnO}, \mathrm{ZnO}$ Shaheed factory and nano $\mathrm{ZnO}$ (Figure 4). The average diameter is $180.79 \mathrm{~nm}, 292.40 \mathrm{~nm}$, and 80 $\mathrm{nm}$ for normal $\mathrm{ZnO}$, shaheed factory $\mathrm{ZnOand}$ nanoZnO respectively.

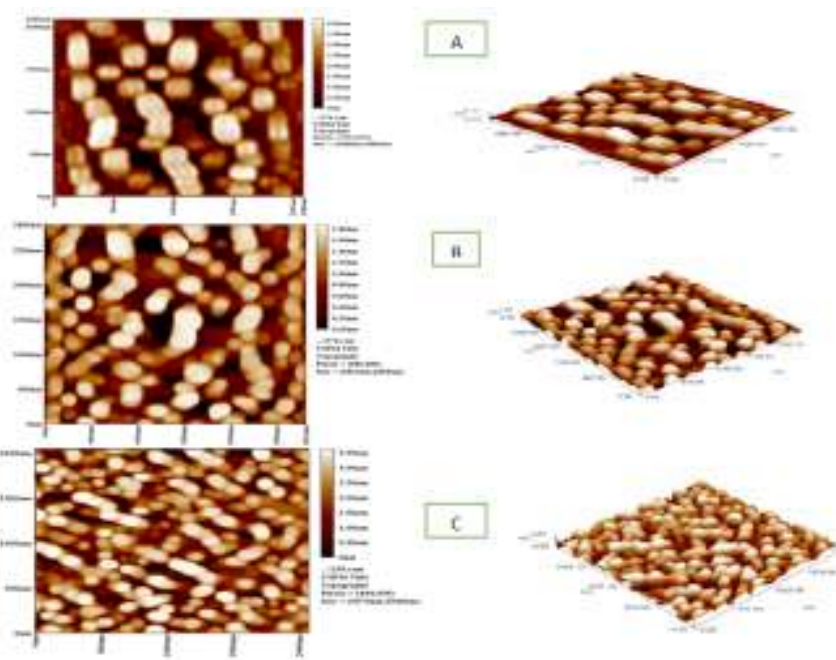

Figure 4: Atomic force microscope image for: A- normal $\mathrm{ZnOB}$ - Shaheed factory $\mathrm{ZnO}, \mathrm{C}$-nanoZnO

\section{2- Adsorption isotherm models:}

Table (1), Figure 5\& Figure 6shows the variation of $\mathrm{Q}_{\mathrm{e}}$ versus $\mathrm{C}_{\mathrm{e}}$ \& different concentration of $\mathrm{M} . \mathrm{B}$ versus \% $\mathrm{R}$ respectively using three types of $\mathrm{ZnO}$ (normal, shaheed, nano) at $298 \mathrm{~K}$.

\begin{tabular}{|c|c|c|c|c|c|c|c|c|c|}
\hline \multicolumn{4}{|c|}{ Normal ZnO } & \multicolumn{3}{|c|}{ ShaheedZnO } & \multicolumn{3}{|c|}{ Nano ZnO } \\
\hline $\mathbf{C}_{0}$ & $\begin{array}{c}\mathrm{C}_{\mathrm{e}} \\
(\mathrm{mg} / \\
\mathrm{ml})\end{array}$ & $\begin{array}{c}Q_{\mathrm{e}} \\
(\mathrm{mg} / \\
\mathrm{g})\end{array}$ & $\begin{array}{l}\% \\
\text { R }\end{array}$ & $\begin{array}{c}\mathrm{C}_{\mathrm{e}} \\
(\mathrm{mg} / \\
\mathrm{ml})\end{array}$ & $\begin{array}{c}Q_{\mathrm{e}} \\
(\mathrm{mg} / \\
\mathrm{g})\end{array}$ & $\begin{array}{l}\% \\
\text { R }\end{array}$ & $\begin{array}{c}\mathrm{C}_{\mathrm{e}} \\
(\mathrm{mg} / \\
\mathrm{ml})\end{array}$ & $\begin{array}{c}Q_{\mathrm{e}} \\
(\mathrm{mg} / \\
\mathrm{g})\end{array}$ & $\begin{array}{l}\% \\
\text { R }\end{array}$ \\
\hline 10 & 2.25 & 4.84 & $\begin{array}{l}77 . \\
50\end{array}$ & 3.51 & 4.05 & $\begin{array}{c}64 . \\
90\end{array}$ & 0.66 & 5.83 & $\begin{array}{l}93 . \\
40\end{array}$ \\
\hline 25 & 9.57 & 9.64 & $\begin{array}{l}61 . \\
72\end{array}$ & 8.52 & 10.3 & $\begin{array}{c}65 . \\
92\end{array}$ & 0.89 & $\begin{array}{c}15.0 \\
6\end{array}$ & $\begin{array}{l}96 . \\
44\end{array}$ \\
\hline 50 & 17.26 & $\begin{array}{c}20.4 \\
6\end{array}$ & $\begin{array}{l}65 . \\
48\end{array}$ & 19.78 & $\begin{array}{c}18.8 \\
8\end{array}$ & $\begin{array}{l}60 . \\
44\end{array}$ & 6.08 & $\begin{array}{c}27.4 \\
5\end{array}$ & $\begin{array}{l}87 . \\
84\end{array}$ \\
\hline $\begin{array}{c}10 \\
0 \\
\end{array}$ & 45.6 & $\begin{array}{c}27.7 \\
5\end{array}$ & $\begin{array}{c}54 . \\
40 \\
\end{array}$ & 48.3 & $\begin{array}{c}29.1 \\
8\end{array}$ & $\begin{array}{c}51 . \\
7\end{array}$ & 25.57 & $\begin{array}{c}46.5 \\
1\end{array}$ & $\begin{array}{l}74 . \\
43\end{array}$ \\
\hline $\begin{array}{c}15 \\
0\end{array}$ & 65 & 53.1 & $\begin{array}{c}56 . \\
6\end{array}$ & 71.79 & $\begin{array}{c}42.6 \\
3\end{array}$ & $\begin{array}{c}52 . \\
14\end{array}$ & 46.67 & $\begin{array}{c}64.5 \\
8\end{array}$ & $\begin{array}{l}68 . \\
88\end{array}$ \\
\hline
\end{tabular}

Table 1: Absorption amount of M.B by different types of $\mathrm{ZnO}$ at $298 \mathrm{~K}$

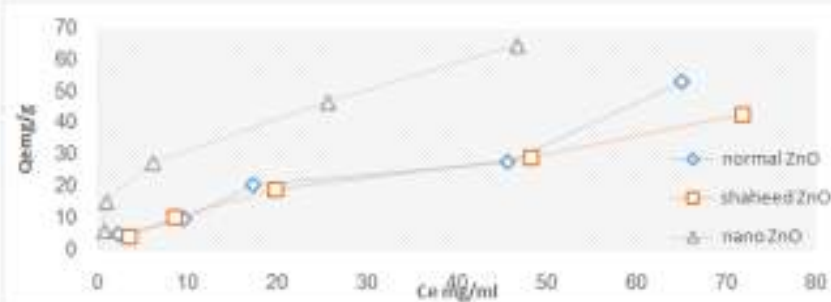

Figure 5: Adsorption isotherm of M.B on: (normal $\mathrm{ZnO}$ ), (shaheed $\mathrm{ZnO}), \&$ (nanoZnO) at $298 \mathrm{~K}$.

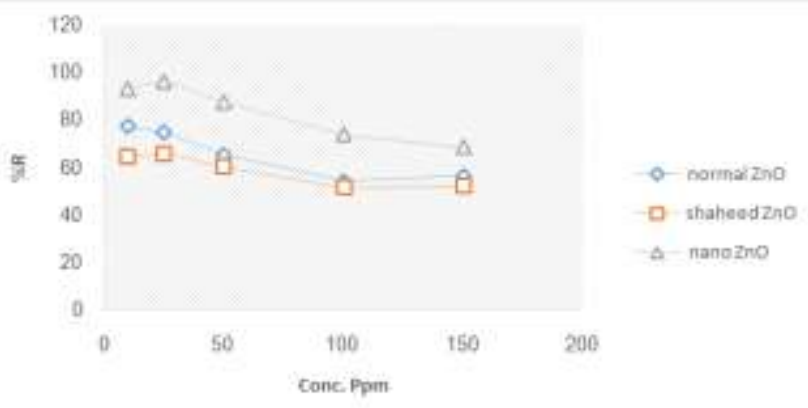

Figure 6: The \%R of M.B on: (normal $\mathrm{ZnO})$, (shaheed $\mathrm{ZnO}) \&($ nanoZnO) at $298 \mathrm{~K}$

It's clear that, the nanoZnO is the best for the removal of M.B than normal $\mathrm{ZnO} \&$ the last compound is the best from shaheed factory $\mathrm{ZnO}$ dependent on particle sized that measured in AFM \& \% R.

Table $2 \&$ Figure 7 show the adsorption parameters for different types of $\mathrm{ZnO}$ at $298 \mathrm{~K}$

Table 2: Absorption value of M.B on different types of $\mathrm{ZnO}$ surfaces for Langmuir application at $298 \mathrm{~K}$

\begin{tabular}{|c|c|c|c|c|c|c|}
\hline \multicolumn{3}{|c|}{ Normal ZnO } & \multicolumn{2}{c|}{ ShaheedZnO } & \multicolumn{2}{c|}{ Nano ZnO } \\
\hline $\mathbf{C}_{\mathbf{0}}$ & $\begin{array}{c}\mathbf{C}_{\mathrm{e}} / \mathbf{q}_{\mathrm{e}} \\
(\mathbf{g} / \mathbf{m l})\end{array}$ & $\begin{array}{c}\mathbf{C}_{\mathrm{e}} \\
(\mathbf{m g} / \mathbf{m l})\end{array}$ & $\begin{array}{c}\mathbf{C}_{\mathrm{e}} / \mathbf{q}_{\mathbf{e}} \\
(\mathbf{g} / \mathbf{m l})\end{array}$ & $\begin{array}{c}\mathbf{C}_{\mathbf{e}} \\
(\mathbf{m g} / \mathbf{m l})\end{array}$ & $\begin{array}{c}\mathbf{C}_{\mathrm{e}} / \mathbf{q}_{\mathbf{e}} \\
(\mathbf{g} / \mathbf{m l})\end{array}$ & $\begin{array}{c}\mathbf{C}_{\mathbf{e}} \\
(\mathbf{m g} / \mathbf{m l})\end{array}$ \\
\hline 10 & 0.46 & 2.25 & 0.86 & 3.51 & 0.11 & 0.66 \\
\hline 25 & 0.99 & 9.57 & 0.82 & 8.52 & 0.05 & 0.89 \\
\hline 50 & 0.84 & 17.26 & 1.04 & 19.78 & 0.22 & 6.08 \\
\hline 100 & 1.64 & 45.6 & 1.65 & 48.3 & 0.54 & 25.57 \\
\hline 150 & 1.22 & 65 & 1.68 & 71.79 & 0.72 & 46.67 \\
\hline
\end{tabular}

Volume 6 Issue 12, December 2017 www.ijsr.net 


\section{International Journal of Science and Research (IJSR) \\ ISSN (Online): 2319-7064}

Index Copernicus Value (2016): 79.57 | Impact Factor (2015): 6.391

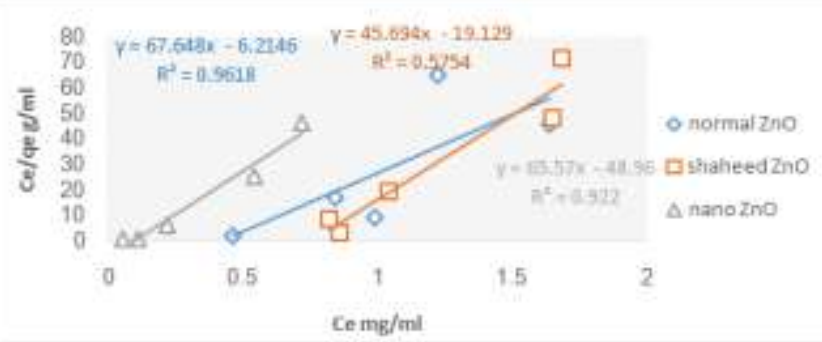

Figure 7: Linear Langmuir Adsorption of M.B dye on: (normal $\mathrm{ZnO}$ ), (shaheedZnO) \& (nanoZnO) at $298 \mathrm{~K}$

Table (3) \& Figure (8) show the Freundlich applied of the adsorption isotherm for M.B on different ZnOtypes at 298K.

Table 3: Absorption value of M.B on different types of $\mathrm{ZnO}$ surfaces for Freundlich application at 298K

\begin{tabular}{|c|c|c|c|c|c|c|}
\hline \multicolumn{3}{|c|}{ Normal ZnO } & \multicolumn{2}{c|}{ ShaheedZnO } & \multicolumn{2}{c|}{ Nano ZnO } \\
\hline $\mathbf{C}_{\mathbf{0}}$ & $\begin{array}{c}\text { Log } \\
\mathbf{Q}_{\mathbf{e}}(\mathbf{m g} / \mathbf{g} \\
)\end{array}$ & $\begin{array}{c}\text { Log C } \\
(\mathbf{m g} / \mathbf{m l} \\
)\end{array}$ & $\begin{array}{c}\text { Log } \\
\mathbf{Q}_{\mathbf{e}}(\mathbf{m g} / \mathbf{g} \\
)\end{array}$ & $\begin{array}{c}\text { Log } \mathbf{C}_{\mathrm{e}} \\
(\mathbf{m g} / \mathbf{m l} \\
)\end{array}$ & $\begin{array}{c}\text { Log } \\
\mathbf{Q}_{\mathbf{e}}(\mathbf{m g} / \mathbf{g} \\
)\end{array}$ & $\begin{array}{c}\mathbf{L o g}_{\mathrm{e}} \\
(\mathbf{m g} / \mathbf{m l} \\
)\end{array}$ \\
\hline 10 & 0.68 & 0.35 & 0.6 & 0.54 & 0.76 & -0.18 \\
\hline 25 & 0.98 & 0.98 & 1.01 & 0.93 & 1.17 & -0.05 \\
\hline 50 & 1.31 & 1.23 & 1.27 & 1.29 & 1.43 & 0.78 \\
\hline $\begin{array}{c}10 \\
0\end{array}$ & 1.44 & 1.74 & 1.46 & 1.72 & 1.66 & 1.4 \\
\hline $\begin{array}{c}15 \\
0\end{array}$ & 1.72 & 1.81 & 1.62 & 1.91 & 1.81 & 1.66 \\
\hline
\end{tabular}

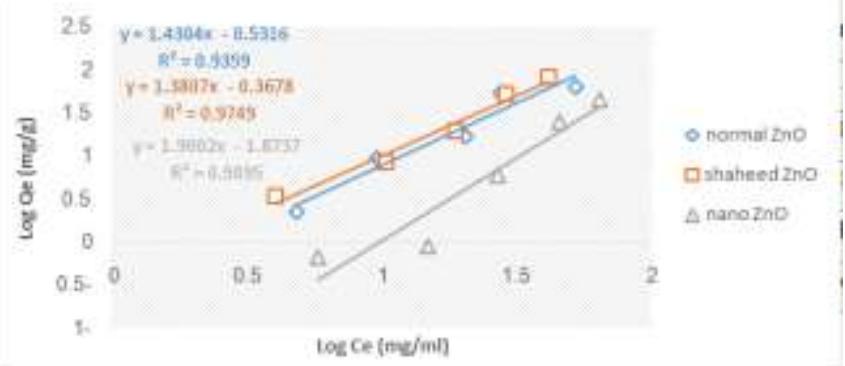

Figure 8: Freundlich Adsorption for M.B dyeon: (normal $\mathrm{ZnO}$ ), (shaheed $\mathrm{ZnO}$ ) \& (nano $\mathrm{ZnO}$ ) at $298 \mathrm{~K}$

Table (4) \& Fig (9) shows the Temkin applied of the adsorption isotherm for M.B on different $\mathrm{ZnO}$ types at $298 \mathrm{~K}$

Table 4: Absorption value of M.B on different types of $\mathrm{ZnO}$ surfaces for Temkin application at $298 \mathrm{~K}$

\begin{tabular}{|c|c|c|c|c|c|c|}
\hline \multicolumn{3}{|c|}{ Normal ZnO } & \multicolumn{2}{|c|}{ ShaheedZnO } & \multicolumn{2}{|c|}{ Nano ZnO } \\
\hline $\mathrm{C}_{\mathrm{o}}$ & $\mathrm{q}_{\mathrm{e}}(\mathrm{mg} / \mathrm{g})$ & $\begin{array}{c}\underset{\mathrm{Ln} \mathrm{C} \mathrm{C}_{\mathrm{e}}}{(\mathrm{mg} / \mathrm{ml})}\end{array}$ & $\mathrm{q}_{\mathrm{e}}(\mathrm{mg} / \mathrm{g})$ & $\begin{array}{c}\mathrm{Ln} \mathrm{C}_{\mathrm{e}} \\
(\mathrm{mg} / \mathrm{ml})\end{array}$ & $q_{e}(\mathrm{mg} / \mathrm{g})$ & $\begin{array}{c}\ln \mathrm{C}_{\mathrm{e}} \\
(\mathrm{mg} / \mathrm{ml})\end{array}$ \\
\hline 10 & 4.84 & 0.81 & 4.05 & 1.25 & 5.83 & -0.41 \\
\hline 25 & 9.64 & 2.25 & 10.3 & 2.14 & 13.06 & -0.11 \\
\hline 50 & 20.46 & 2.84 & 18.88 & 2.98 & 27.45 & 1.8 \\
\hline 100 & 27.75 & 4.01 & 29.18 & 3.97 & 46.51 & 3.24 \\
\hline 150 & 53.1 & 4.17 & 42.63 & 4.4 & 64.58 & 3.84 \\
\hline
\end{tabular}

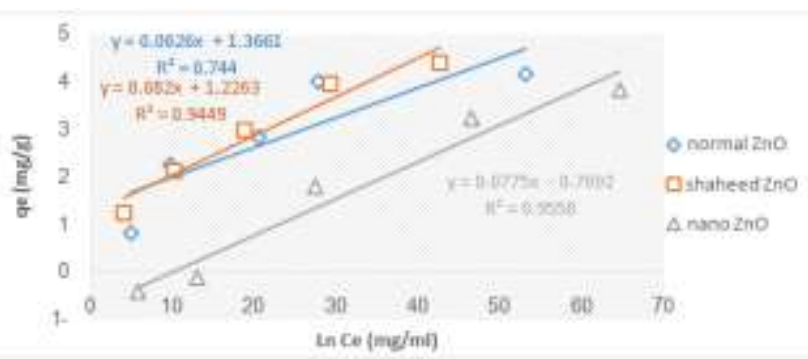

Figure 9: Temkin Adsorption for M.B dyeon: (normal $\mathrm{ZnO}),($ shaheedZnO) \& (nanoZnO ) at $298 \mathrm{~K}$

Table (5) shows the empirical values \& correlation factors for Langmuir, Freundlich \& temkin constants.

Table 5: Empirical values \& correlation factors for Langmuir, Freundlich \& Temkin constants for the adsorption of M.B by different types $\mathrm{ZnO}$ at $298 \mathrm{~K}$

\begin{tabular}{|c|c|c|c|c|}
\hline $\begin{array}{c}\text { Isotherm } \\
\text { models }\end{array}$ & parameters & $\begin{array}{c}\text { Normal } \\
\mathrm{ZnO} \text { at } \\
\text { temperature }\end{array}$ & $\begin{array}{c}\text { shaheedZnO } \\
\text { at } \\
\text { temperature }\end{array}$ & $\begin{array}{c}\text { nanoZnO at } \\
\text { temperature }\end{array}$ \\
\hline \multirow{3}{*}{ Langmuir } & $\mathrm{q}_{\mathrm{m}}\left(\mathrm{mgg}^{-1}\right)$ & 79.63 & 70.92 & 70.42 \\
\cline { 2 - 5 } & $\mathrm{K}_{\mathrm{L}\left(\mathrm{Lmg}^{-1}\right)}$ & 0.0185 & 0.0180 & 0.1407 \\
\cline { 2 - 5 } Freundlich & $\mathrm{R}^{2}$ & 0.5754 & 0.9220 & 0.9618 \\
\cline { 2 - 5 } & $\mathrm{K}_{\mathrm{F}}\left(\mathrm{lmg}^{-1}\right)$ & 2.669 & 2.332 & 74.765 \\
\cline { 2 - 5 } & $1 / \mathrm{n}$ & 0.654 & 1.381 & 1.901 \\
\hline \multirow{3}{*}{ Temkin } & $\mathrm{R}^{2}$ & 0.9395 & 0.9749 & 0.9095 \\
\cline { 2 - 5 } & $\mathrm{A}_{\mathrm{T}}(\mathrm{L} / \mathrm{g})$ & 2.380 & 3.650 & 2.415 \\
\cline { 2 - 5 } & $\mathrm{B}\left(\mathrm{J} / \mathrm{mol}^{2}\right)$ & 11.883 & 11.529 & 12.328 \\
\cline { 2 - 5 } & $\mathrm{R}^{2}$ & 0.7440 & 0.9449 & 0.9558 \\
\hline
\end{tabular}

The normal $\mathrm{ZnO} \&$ shaheed $\mathrm{ZnO}$ were fitting to Freundlich equation. The best fit of equilibrium data that on the (Freundlich isotherm) given a heterogeneous surface with nonuniform distribution of heat of adsorption over the surface. The value of $1 / \mathrm{n}$ for (normal \&nano ) $\mathrm{ZnO}$ referred to physisorption while the adsorption on shaheed $\mathrm{ZnO}$ (chemisorption) \& correlation coefficient $\left(\mathrm{R}^{2}\right)$ indicated that the M.B sorption process was favorable to Freundlich model [27], the value of $1 / \mathrm{n}(0.1<1 / \mathrm{n}<1)$ referred to favorable adsorption of M.B dye at experimental condition while the adsorption data for M.B with nanoZnO was fitting to Temkinisotherm model dependent on the $\mathrm{R}^{2}$ value that referred to the M.B adsorption is characterized by a uniform distribution of binding energies, up to some maximum binding energy[28].

\section{3- Thermodynamic parameters:}

Thermodynamic parameters derived from equation (12) for M.B adsorption ( $25 \mathrm{ppm}$ on different $\mathrm{ZnO}$ types is shown in the table (8). 


\section{International Journal of Science and Research (IJSR) \\ ISSN (Online): 2319-7064}

Index Copernicus Value (2016): 79.57 | Impact Factor (2015): 6.391

Table 8: Comparison of thermodynamic parameters for adsorption of $25 \mathrm{ppm}$ M.B dye on different types of $\mathrm{ZnO}$

\begin{tabular}{|c|c|c|c|c|c|c|}
\hline $\begin{array}{c}\mathrm{T}(\mathrm{K} \\
)\end{array}$ & $\underset{1}{1 / \mathrm{T}}\left(\mathrm{K}^{-}\right.$ & $\mathrm{K}_{\mathrm{ZnO} \text { normal }}$ & Ln $\mathrm{K}$ & $\begin{array}{c}\Delta \mathrm{G}^{\mathrm{o} \mathrm{kJ}} / \mathrm{mo} \\
1\end{array}$ & $\begin{array}{c}\Delta \mathrm{H}^{\mathrm{o}} \\
\mathrm{kJ} / \mathrm{mo} \\
1\end{array}$ & $\begin{array}{c}\Delta \mathrm{S}^{\circ} \mathrm{J} / \mathrm{mol} \\
\mathrm{K}\end{array}$ \\
\hline 293 & 0.0034 & 4.470 & $\begin{array}{c}1.49 \\
7\end{array}$ & -3.64 & \multirow{4}{*}{-11.09} & \multirow{4}{*}{-25.38} \\
\hline 298 & $\begin{array}{c}0.0033 \\
5\end{array}$ & 1.612 & $\begin{array}{c}1.28 \\
4\end{array}$ & -1.18 & & \\
\hline 303 & 0.0033 & 3.424 & $\begin{array}{c}1.23 \\
1\end{array}$ & -3.1 & & \\
\hline 308 & 0.0032 & 3.307 & $\begin{array}{c}1.19 \\
6 \\
\end{array}$ & -3.06 & & \\
\hline $\begin{array}{c}\mathrm{T}(\mathrm{K} \\
)\end{array}$ & $\begin{array}{c}1 / \mathrm{T} \\
\left.{ }^{1}\right)\end{array}$ & $\begin{array}{c}\mathrm{K}_{\mathrm{ZnOshahee}} \\
\mathrm{d}\end{array}$ & Ln K & $\begin{array}{c}\Delta \mathrm{G}^{\mathrm{o} \mathrm{kJ}} / \mathrm{mo} \\
1\end{array}$ & $\begin{array}{c}\Delta \mathrm{H}^{\mathrm{o}} \\
\mathrm{kJ} / \mathrm{mo} \\
1\end{array}$ & $\begin{array}{c}\Delta \mathrm{S}^{\circ} \mathrm{J} / \mathrm{mol} . \\
\mathrm{K}\end{array}$ \\
\hline 293 & 0.0034 & 1.312 & $\begin{array}{c}0.27 \\
2\end{array}$ & -0.66 & \multirow{4}{*}{14.28} & \multirow{4}{*}{51.96} \\
\hline 298 & $\begin{array}{c}0.0033 \\
5\end{array}$ & 1.934 & $\begin{array}{c}0.65 \\
9\end{array}$ & -1.63 & & \\
\hline 303 & 0.0033 & 1.834 & $\begin{array}{c}0.60 \\
6\end{array}$ & -1.52 & & \\
\hline 308 & 0.0032 & 2.011 & $\begin{array}{c}0.69 \\
8 \\
\end{array}$ & -1.78 & & \\
\hline $\begin{array}{c}\mathrm{T}(\mathrm{K} \\
)\end{array}$ & $\underset{1}{1 / \mathrm{T}}\left(\mathrm{K}^{-}\right.$ & $\mathrm{K}_{\mathrm{ZnOnano}}$ & Ln K & $\begin{array}{c}\Delta \mathrm{G}^{\mathrm{o}} \mathrm{kJ} / \mathrm{mo} \\
1\end{array}$ & $\begin{array}{c}\Delta \mathrm{H}^{\mathrm{o}} \\
\mathrm{kJ} / \mathrm{mo} \\
1\end{array}$ & $\begin{array}{c}\Delta \mathrm{S}^{\circ} \mathrm{J} / \mathrm{mol} \\
\mathrm{K}\end{array}$ \\
\hline 293 & 0.0034 & 40.05 & 3.69 & -8.98 & \multirow{4}{*}{-36.71} & \multirow{4}{*}{-93.25} \\
\hline 298 & $\begin{array}{c}0.0033 \\
5 \\
\end{array}$ & 27.08 & $\begin{array}{c}3.29 \\
9\end{array}$ & -8.17 & & \\
\hline 303 & 0.0033 & 53.94 & $\begin{array}{c}3.98 \\
7\end{array}$ & -10.04 & & \\
\hline 308 & 0.0032 & 14.42 & $\begin{array}{c}2.66 \\
8\end{array}$ & -6.83 & & \\
\hline
\end{tabular}

It clear that, the adsorption of M.B on different $\mathrm{ZnO}$ types negative value $\Delta \mathrm{G}^{\mathrm{o}}$ indicated to the adsorption process was spontaneously and thermodynamic favorable. The negative value of free energy with increase temperature indicated to the driving force at adsorption process. The adsorption process with (nano \& normal) $\mathrm{ZnO}$ were exothermic dependent on the value of $\Delta \mathrm{H}^{\mathrm{o}}$ (negative), while the $\Delta \mathrm{H}^{\mathrm{o}}$ values of $\mathrm{M}$.B adsorption on shaheed $\mathrm{ZnO}$ have positive sign reflecting on endothermic process. The value of $\Delta \mathrm{S}^{\circ}$ with (nano \& normal) $\mathrm{ZnO}$ were negative which referred to decrease disorder at the solid/liquid interface during M.B sorption while the $\Delta \mathrm{S}^{\mathrm{o}}$ for shaheed $\mathrm{ZnO}$ was positive which referred to increase disorder at the solid/liquid interface during M.B sorption [29].

\section{Conclusion}

1. Methylene blue dye have an aromatic structure, substituted with ionic part $\left(\mathrm{Cl}^{-}\right)$, this part can be connected with $\mathrm{ZnO}$ surface by sorption process. Therefore, can be remove the M.B dye.

2. The sorption of M.B dye on (normal, shaheed) $\mathrm{ZnO}$ was of Freundlich type, \& this indicates that multi-layer has formed.

3. The sorption of M.B dye on nano $\mathrm{ZnO}$ was of Temkin process \& this indicates that decrease linearity.

4. When the average diameter was decrease, the adsorption for M.B increase.

5. The negative value of $\Delta \mathrm{H}$ indicates that the adsorption process is dominating rather than M.B may also inter inside the $\mathrm{ZnO}$ surface.

\section{References}

[1] Mekkawy, H. A., M. O. Ali, and A. M. El-Zawahry. "Toxic effect of synthetic and natural food dyes on renal and hepatic functions in rats." Toxicology Letters 95 (1998): 155.

[2] Nordberg, Erik, and Uno Winblad. "Environmental hygiene in Sida-supported programmes in Africa: review and recommendations." Environmental hygiene in SIDA-supported programmes in Africa: review and recommendations. Swedish International Development Authority, 1990.

[3] Oz, Murat, et al. "Cellular and molecular actions of methylene blue in the nervous system." Medicinal research reviews 31.1 (2011): 93-117.

[4] Ruggaber, Timothy P., and Jeffrey W. Talley. "Enhancing bioremediation with enzymatic processes: a review." Practice Periodical of Hazardous, Toxic, and Radioactive Waste Management 10.2 (2006): 73-85

[5] Duran, Nelson, and Elisa Esposito. "Potential applications of oxidative enzymes and phenoloxidase-like compounds in wastewater and soil treatment: a review." Applied catalysis B: environmental 28.2 (2000): 83-99.

[6] Yang, Chufen, et al. "Solvent extraction process development and on-site trial-plant for phenol removal from industrial coal-gasification wastewater." Chemical Engineering Journal 117.2 (2006): 179-185.

[7] Yousef, Rushdi I., and Bassam El-Eswed. "Adsorption behavior of chlorophenols on natural zeolite." Separation Science and Technology 42.14 (2007): 3187-3197.

[8] Wang, G. S., et al. "Characteristics of natural organic matter degradation in water by UV/H2O2 treatment." Environmental technology 27.3 (2006): 277-287.

[9] Chen, Guohua. "Electrochemical technologies in wastewater treatment." Separation and purification Technology 38.1 (2004): 11-41

[10] Kidak, R., and N. H. Ince. "Catalysis of advanced oxidation reactions by ultrasound: A case study with phenol." Journal of Hazardous Materials 146.3 (2007): 630-635.

[11] Y. K. Al-Bayati and R.R.Karabat, potentiometric sudy of phenytoin -pvc membrane electrodes for determination of phenytoin in pharmaceutical preparations, Journal of Al-Nahrain University (2015).

[12] Sing, K. S. W. "Adsorption, Surface Area and Porosity." Academic Press, London (1982): 19-32.

[13] Parfitt, Geoffrey D., and Colin H. Rochester, eds. Adsorption from solution at the solid/liquid interface. Vol. 3. London: Academic press, 1983.

[14] W.J.thomson., "Adsorption Technology", Butterworth, London, (1983).

[15] http:

//www.micromeritics.com/Repository/Files/Chemis orption_Poster.pdfJ.O.Oscik., and I.L. Cooper., "Adsorption", 15 ${ }^{\text {th }}$ Ed., Jonhn Wiley and Sons, New York., (1982). 


\section{International Journal of Science and Research (IJSR) \\ ISSN (Online): 2319-7064}

Index Copernicus Value (2016): 79.57 | Impact Factor (2015): 6.391

[16] Dada, A. O., et al. "Langmuir, Freundlich, Temkin and Dubinin-Radushkevich isotherms studies of equilibrium sorption of $\mathrm{Zn} 2+$ unto phosphoric acid modified rice husk." IOSR Journal of Applied Chemistry 3.1 (2012): 38-45.

[17] Langmuir, Irving. "The adsorption of gases on plane surfaces of glass, mica and platinum." Journal of the American Chemical society40.9 (1918): 13611403.

[18] Boparai, Hardiljeet K., Meera Joseph, and Denis M. O'Carroll. "Kinetics and thermodynamics of cadmium ion removal by adsorption onto nanozerovalent iron particles." Journal of hazardous materials 186.1 (2011): 458-465.

[19] Voudrias, E., K. Fytianos, and E. Bozani. "Sorption-desorption isotherms of dyes from aqueous solutions and wastewaters with different sorbent materials." Global Nest Int J 4 (2002): 7583.

[20] Freundlich, H., Heller, W., 1939. The adsorption of cis- and trans- azobenzene. J. Am. Chem. Soc. 61, 2228-2230.

[21] Tempkin, M. I., and V. Pyzhev. "Kinetics of ammonia synthesis on promoted iron catalyst." Acta Phys. Chim. USSR 12.1 (1940): 327.

[22] Aharoni, Chaim, and Moshe Ungarish. "Kinetics of activated chemisorption. Part 2.-Theoretical models." Journal of the Chemical Society, Faraday Transactions 1: Physical Chemistry in Condensed Phases 73 (1977): 456-464.

[23] ALzaydien, Atef S. "Adsorption of methylene blue from aqueous solution onto a low-cost natural Jordanian Tripoli." American Journal of Applied Sciences 6.6 (2009): 1047.

[24] Akyol, A., and M. Bayramoğlu. "Photocatalytic degradation of Remazol Red F3B using $\mathrm{ZnO}$ catalyst." Journal of hazardous materials 124.1 (2005): 241-246.

[25] AbdElghafar, M.A.; Youssef, A.M.; and AbdElhakim, A.A. "Polyanalinenanocomposites via in situemlsion polymerization based on montmorillonite: preparation and characterization". Arabian journal of chemistry, available online(2014) 17 January.

[26] Li, Q., Yue, Q., Su, Y., Gao, B., Sun, H., 2010. Equilibrium, thermodynamics and process design to minimize adsorbent amountfor the adsorption of acid dyes onto cationic polymer-loadedbentonite. Chem. Eng. J. 158, 489-497.

[27] Elkady, M. F., Amal M. Ibrahim, and MM Abd ElLatif. "Assessment of the adsorption kinetics, equilibrium and thermodynamic for the potential removal of reactive red dye using eggshell biocomposite beads." Desalination 278.1 (2011): 412-423.

[28] Temkin, M. J., and V. Pyzhev. "Recent modifications to Langmuir isotherms." (1940): 217222.

[29] Zelentsov, Veaceslav, and Tatiana Datsko. "Thermodynamics of fluorine adsorption onto modified Trepel." Termotehnica 1 (2013): 25-30

Volume 6 Issue 12, December 2017 www.ijsr.net 